\title{
Acoustic Resonance Testing of Glass IV Bottles
}

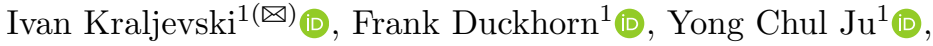 \\ Constanze Tschoepe ${ }^{1}\left(\mathbb{D}\right.$, and Matthias Wolff ${ }^{2}(\mathbb{D}$ \\ 1 Fraunhofer Institute for Ceramic Technologies and Systems IKTS, \\ Dresden, Germany \\ \{ivan.kraljevski,frank.duckhorn, yong.chul.ju, \\ constanze.tschoepe\}@ikts.fraunhofer.de \\ 2 Chair of Communications Engineering, Brandenburg University of Technology \\ (BTU) Cottbus-Senftenberg, Cottbus, Germany \\ matthias.wolff@b-tu.de
}

\begin{abstract}
In this paper, acoustic resonance testing on glass intravenous (IV) bottles is presented. Different machine learning methods were applied to distinguish acoustic observations of bottles with defects from the intact ones. Due to the very limited amount of available specimens, the question arises whether the deep learning methods can achieve similar or even better detection performance compared with traditional methods.

The results from the binary classification experiments are presented and compared in terms of Balanced Accuracy Rate, F1-score, Area Under the Receiver Operating Characteristic Curve and Matthews Correlation Coefficient metrics.

The presented feature analysis and the employed classifiers achieved solid results, despite the rather small and imbalanced dataset with a highly inconsistent class population.
\end{abstract}

Keywords: Acoustic resonance testing - Machine learning - Glass IV bottles $\cdot$ Non-destructive testing

\section{Introduction}

Glass materials are ubiquitous in many areas of everyday life, in the home, industry, medicine, vehicles, etc. The production quality of glass is of the highest importance, however, defects in glass materials may occur in the form of cracks, spots, bubbles and inclusions, holes and abrasions [19].

Different defects will have different impacts on the usage of glass products, while cracks may have little effect on the ordinary household glass, even hairline or micro-sized cracks will have large effects on the glassware used in the pharmaceutical and medical industries. 
Therefore, it is necessary to detect the presence of any defects in glass materials by employing non-destructive testing (NDT) in fabrication and in-service inspections to ensure product integrity and reliability.

Common approaches to NDT of glass materials are X-ray Computer Tomography $(\mathrm{XCT})$, optical systems (OS) and acoustic emission testing (AE) [1]. In production, machine vision-based systems are providing reliable defect detection [18], while in-service it would be difficult to deploy equipment and trained operators to track defects and subjectively interpret results. Many studies present such systems $[14,21,26,32]$, where image processing algorithms are used to detect glassware defects.

To the best of our knowledge, machine learning (ML) approaches have not been extensively applied in the field of NDT on glassware and there are fewer studies on it. In one of them [15], the authors applied Convolutional Neural Networks (CNNs) on images to detect defects in the mouth, body and the bottom of glass bottles achieving an average accuracy rate of $98.4 \%$.

Alternatives to image-based systems are Resonance Acoustic Method (RAM) [25], known as Acoustic Resonance Testing (ART) [7] NDT systems.

The mechanical vibrations in the structure are produced by impact and transmitted as audible signals carrying information about the object's material, structure and geometry in their entirety, but alone it will generally not diagnose the location, size, or type of defect.

ART was successfully applied in many areas, such as in automotive production lines [12]. Artificial Neural Networks (ANN) were used for classifying automotive components as intact, without damage or defective with results that completely prevented false positives and only $2.61 \%$ of good parts detected as defective [23]. Classification of magnetic tiles (qualified/unqualified) by their acoustic resonance after impact with a metal block was presented in [30].

Impact tests to determine defects in glassware using Fourier analysis of the resonance frequency are presented in [11]. Wavelet packet transforms and ANNs were used for the same problem in [10], achieving an accuracy rate of $96.6 \%$ on 3 classes (no glass, good, defective). Smart signal processing in ART is crucial since every defect will produce a corresponding dynamic response depending on its size and the feature analysis should be able to reliably capture the deviations.

In this paper, acoustic resonance testing of glass IV (intravenous) bottles is presented. We collected a small database in trials where a hammer impact was used as excitation. The observation signals were labeled, pre-processed and feature analysis was performed. Different ML approaches were applied, and the results of the binary classification were presented and compared. Since the amount of the collected data is small, the question arises whether deep learning could provide comparable or even better performance against traditional ML methods.

The paper is organized as follows: Sect. 2 describes the selection of glass IV bottles used in the experiments, as well as, the data collection and their organization in a dataset and the employed feature analysis algorithms. 
In Sect.3, we first present the experimental setup and then each of the used ML approaches with their specific parameter configurations. In Sect. 4 we present the achieved results in the classification experiments across different machine learning and feature analysis approaches. We close this paper with concluding remarks in Sect. 5 .

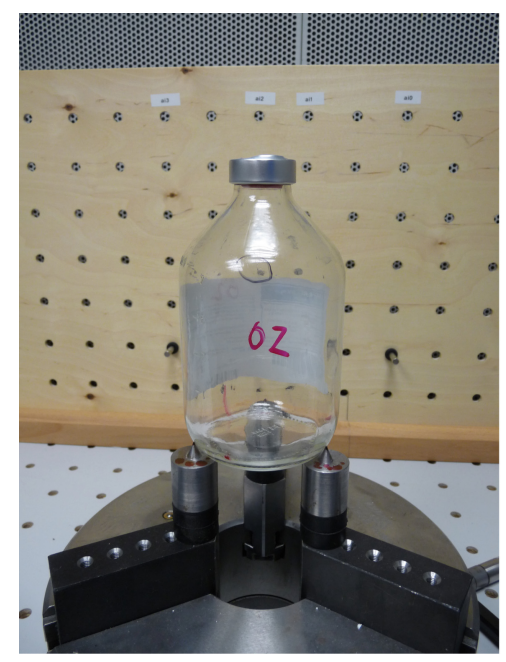

(a) Specimen B2

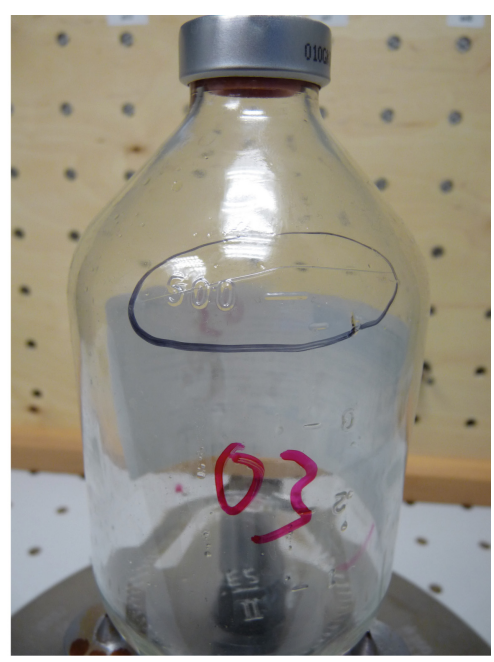

(b) Specimen B3

Fig. 1. Recording setup with naturally damaged bottles

\section{Materials and Methods}

The selection of glass IV bottles consists of used and empty ones which are: intact (120), artificially (10) and naturally (3) damaged.

To provide representative examples of defects, different types of damages were artificially introduced to intact bottles in the form of smaller and larger cracks, abrasions and holes. In this case, each specimen has characteristic damage of different sizes and placement.

Bottles with defects that occurred naturally have small (the specimens B1 and B2) to middle size (the specimen B3) hairline cracks on the glass surface.

The bottles have the used rubber cap still on, and there was some intravenous fluid still present in most of them, which influenced their physical structure and consequently defined a unique set of characteristic spectral and temporal features, e.g. vibration properties. 


\subsection{Data Collection and Organization}

The recording sessions were carried out in a soundproof room, where the acoustic responses of the bottles were measured by a microphone array [28].

A human operator was knocking the bottles using a modal hammer on three different locations, a force sensor was used as a recording trigger, ensuring consistent acoustic signal onset across the recordings. The recording setup with two different damaged bottles with marked cracks is presented in Fig. 1.

The acoustic responses were acquired by four high-performance Microtech Gefell MK 301 E microphone cartridges placed horizontally at: -125, - 25, 25 and $125 \mathrm{~mm}$ of the field center, and $125 \mathrm{~mm}$ vertically from the base. The bottles were placed at a distance of $30 \mathrm{~cm}$ and a height of $165 \mathrm{~mm}$, the holder was aligned by a perforated base plate with the microphone field in the center. The hammer impacts were applied at the positions (20 times on each): 0, 120 and 240 degrees counter-clockwise rotation, the 0 degree position is where the bottle label is aligned with a marked holder.

The dataset contains 8002 observations labeled according to the condition of the IV bottle specimens. The originally damaged bottles were labeled with "B" (broken) - 181 (2.3\%), the artificially damaged with "D" (defective) - 602 $(7.5 \%)$ and the undamaged bottles were labeled with "V" (valid) $7219(90.2 \%)$ observations.

\section{$2.2 \quad$ Feature Analysis}

Acoustic response observations were obtained by simple delay-and-sum beamforming of individual microphone signals, providing more acoustic information than separate ones, at the same time reducing the computational costs of the feature analysis and the classification.

The acoustic resonance observations were transformed into three different feature sets:

1. The raw signal (SIG) observations, where the analog signals were A/D converted to $48 \mathrm{kHz}, 16$ bit, little-endian PCM and summed into one single observation with a fixed duration of one second, the dimension of the features is $48000 \times 1$ elements.

2. The primary feature analysis (PFA) was done over the SIG features, by short-time Fourier transformation with a Blackman window of 512, and MEL filter-bank with a triangular transfer function. The feature vector dimension per observation is $298 \times 30$.

3. Secondary feature analysis (SFA) was performed over the PFA features by their standardization to zero mean and unit variance, computing the delta features and performing Principal Component Analysis (PCA) for size reduction, producing feature vectors with a dimension of $298 \times 24$. 


\section{$3 \quad$ Experiments and Results}

The choice of an appropriate machine learning approach should also be based on the following factors:

- tolerance of high dimensionality,

- the capability of exploiting a small dataset, and

- handling of imbalanced datasets.

The objective is to find out which ML approach is best suited for the given dataset: Hidden Markov Models (HMMs), Support Vector Machine (SVM) models or Deep Learning methods.

Stratified 5-fold Cross-Validation (CV) was employed in all experiments, ensuring proper representations of the classes in the train and the test folds. The 95\% confidence intervals (CI) for balanced accuracy rate (BAR) [3], the macro average $(\mu)$ and standard deviation $(\sigma)$ of the F1 score were used as performance metrics. The Area Under the Curve (AUC) and the Matthews Correlation Coefficient (MCC) were calculated, as well. The class weights were applied accordingly in the training procedure to anticipate the highly imbalanced dataset during training.

The objective of Experiment 1 is to classify acoustic responses of intact and artificial damaged bottles. The dataset folds were created by random sampling at the same time ensuring that the train and the test sets do not contain the same signal observations. The class distribution was also taken into account and the classes were accordingly represented in both sets.

In the second experiment (Experiment 2), the training was performed by leaving out a specific group of bottles from the $\mathrm{k}$-fold training runs. They were used as an additional dataset and tested on the models trained for each fold.

This represents a real use-case, where few damaged specimens are available. In the case when no damaged specimens are available at all, other ML approaches for one-class or anomaly (outliers) detection have to be considered.

Here, the dataset was divided into disjoint groups: V1, V2, and V3 (each with different 40 bottles from class V), B1, B2, and B3 (each with one bottle from class B). The training was performed in the following combinations:

- training V1, V2, B1, B2, and test V3, B3;

- training V1, V3, B1, B3, and test V2, B2;

- training V2, V3, B2, B3, and test V1, B1.

The false negative rates (FNR) of the observations of natural damaged bottles ("B") with the $95 \%$ CI were calculated. Miss-classifying observations of damaged bottles (positive identification) as observations of intact ones is more critical than the miss-classifying observations of intact bottles as damaged (false positives).

\subsection{Hidden Markov Models}

Hidden Markov Models (HMM), model the signals as Markov processes where the states emit the observations by a Gaussian probability density function. 
Apart from other areas of application (speech and handwriting recognition, bioinformatics, etc.) they were also successfully applied in NDT [27].

In the experiments, the HMMs were created using the dLabPro software $[13,29]$. To represent the subtle temporal features, the classes were modeled by five-state forward connected HMMs with exactly one Gaussian probability density function (PDF) and full covariance matrix per state. The model parameters are iteratively estimated by the Viterbi training algorithm alternated with Gaussian splitting, with maximum of 5 splits and a different number of iterations per split.

The similarity measure describes how well a model fits an observation and is estimated by computing neg-log likelihoods (NLLs). The model which yields the highest likelihood defined predicted class label.

\subsection{Support Vector Machines}

SVMs predict class labels by finding the best hyperplane that separates samples of one class from those of the other class. They are very effective in the case where the dimension of the features is much higher than the number of observations in the dataset. The choice of kernel parameters plays an important role in achieving acceptable results for binary classification with SVMs [8].

To train the SVM models, we used the python scikit-learn [20] interface to the well known LIBSVM library [4]. The Radial Basis Function (RBF) was chosen as a kernel because of its good general performance and the SVMs were tuned over a range of the cost $\left(10^{-4}\right.$ to $\left.10^{1}\right)$ and the gamma $\left(10^{-9}\right.$ to $\left.10^{1}\right)$ parameters.

\subsection{Deep Neural Networks}

Deep Feed-forward (DFFN) and Convolutional Neural Network (CNN) were trained over the feature sets with Keras [5] and Tensorflow [17]. For all employed classifiers random search was performed over the hyper-parameter space in the pre-tests to estimate appropriate architectures and parameter values.

The DFFN architectures consist of an input layer corresponding to the feature vectors, output layer with one unit and softmax activation. They have two fully connected hidden layers: the first with 256 (128 for SFA features) units and the second with the half of units from the first layer, respectively.

The Leaky Rectified Linear Unit (LeakyReLU) [16] was used as the activation function, batch normalization and dropout layers [24] with Adaptive Gradient Algorithm (Adagrad) [9] as the optimization function.

For PFA and SFA features, the CNN architectures have two 2D convolutional layers with 32 filters of size $5 \times 5$, Exponential Linear Unit activation (ELU) [6], followed by batch normalization, dropout, and MaxPooling layers.

The output of the convolutional layers was flattened and feed to a fully connected layer with 90 units and ReLU activation and Adadelta [31] as optimization function. 
One dimensional CNN based on the Soundnet architecture [2] was used for the SIG features (Table 1), with Rectified Linear Unit (ReLU) activation, batch normalization, dropout and MaxPooling layers (MPL).

Table 1. The configuration of the convolutional and pooling layers applied to raw audio signals.

\begin{tabular}{|c|c|c|c|c|c|c|c|c|}
\hline & \multicolumn{8}{|c|}{ Layers } \\
\hline & CL1 & MPL1 & CL2 & MPL2 & CL3 & CL4 & CL5 & MPL3 \\
\hline Dim. & 24000 & 3000 & 1500 & 188 & 24 & 12 & 6 & 2 \\
\hline \# Filters & 16 & 16 & 32 & 32 & 64 & 128 & 256 & 256 \\
\hline Filter size & 64 & 8 & 32 & 8 & 16 & 8 & 4 & 4 \\
\hline Stride & 2 & 8 & 2 & 8 & 2 & 2 & 2 & 4 \\
\hline
\end{tabular}

The output of the convolution layers (with a dimension of 512) was flattened into two fully connected layers with 64 and 32 units respectively, providing a rather small size of trainable parameters (283217) well suited for small datasets. For all of them, the output layer has a softmax activation and binary-cross entropy as a loss function.

To avoid over-fitting which is even more emphasized in the case of a small dataset and larger feature dimensions, proper parameters for optimizer algorithms, training batch size, dropout rate, and early stopping criteria, were discovered during hyperparameter optimization.

The maximum number of epochs was set to 200 with the condition of 10 (DFFNs) or 25 (CNNs) epochs with no improvement (patience parameter) after which the training was stopped. The patience parameter and the high dropout rate allow longer training and better generalization.

\section{Results and Discussion}

The inconsistency of the dataset samples across classes was the main issue as already described in Sect. 2. This influenced the overall resonance frequencies and could mask those related to the condition of the bottle.

Also, the hammer impacts made by a human operator gave inconsistent acoustic resonance responses per specimen. On the other hand, this represents the real use-case, where inexperienced personnel should assess the glassware condition in a non-ideal environment.

\subsection{Experiment 1}

Table 2 presents the results achieved in Experiment 1, in terms of balanced accuracy rate $(\mathrm{BAR})$ with the $95 \%$ confidence intervals and the mean $(\mu)$ of the $\mathrm{F} 1$ scores across folds with standard deviation $(\sigma)$, across all features and classifiers. 
Table 2. Balanced Accuracy Rate (\%) and F1-score.

\begin{tabular}{l|l|l|l}
\hline Experiment 1 & SIG & PFA & SFA \\
\hline 95\% CI of BAR & \multicolumn{3}{l}{} \\
\hline HMM & N/A & $91.17_{-0.94}^{+0.86}$ & $93.90_{-0.78}^{+0.67}$ \\
\hline SVM & $89.45_{-1.02}^{+0.93}$ & $90.85_{-1.39}^{+1.27}$ & $84.75_{-1.62}^{+1.58}$ \\
\hline DFFN & $60.66_{-1.49}^{+1.51}$ & $82.36_{-1.68}^{+1.62}$ & $80.80_{-1.72}^{+1.68}$ \\
\hline CNN & $86.60_{-1.53}^{+1.47}$ & $93.05_{-1.23}^{+1.12}$ & $88.50_{-1.47}^{+1.43}$ \\
\hline F1-score $: \mu(\sigma)$ & N/A & $0.77(0.01)$ & $0.83(0.02)$ \\
\hline HMM & $0.74(0.01)$ & $0.93(0.01)$ & $0.89(0.01)$ \\
\hline SVM & $0.63(0.04)$ & $0.86(0.02)$ & $0.84(0.02)$ \\
\hline DFFN & $0.87(0.02)$ & $0.94(0.01)$ & $0.90(0.02)$ \\
\hline CNN &
\end{tabular}

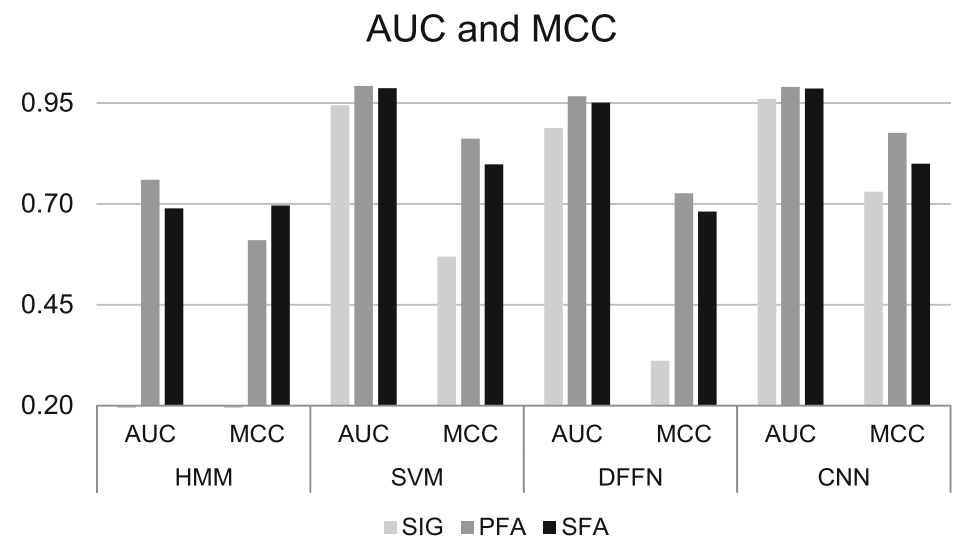

Fig. 2. AUC and MCC across features and classifiers.

The SIG/HMM combination was omitted from the experiments as infeasible due to the dimensionality of the features.

It can be seen that CNN models performed well across all feature types in terms of BAR and better than any other in terms of F1-score, despite small and imbalanced dataset. The models were able to discover patterns that can be interpreted as detectors of dynamic events in the time domain (SIG), as well as, in the time-frequency domain (PFA and SFA). The PFA features have the edge over the raw signals for all classifiers except HMMs, mainly because of the dimensionality reduction which compensates the smaller observation counts.

In the pre-trials with even a smaller dataset (600 samples), the performance gap between the raw signal-based features and the other two for each classifier was even larger. Given more training data, it is expected that CNNs outperform 
the other approaches on raw signals, where more optimal that the handcrafted features will be discovered.

Figure 2 presents the AUC and the MCC metrics for the binary classification, where the damaged bottle label was chosen as positive, since detecting those observations correctly is more important than the intact ones.

The CNNs are performing equally well as the SVMs which, in general, are more suitable choice for binary classification given the size and nature (imbalanced data) of the dataset.

\subsection{Experiment 2}

Because they are of higher importance, Table 3 presents only the results for false negative rates on class "B" obtained in Experiment 2. It can be seen that most of the classifier/feature models failed to reliably detect unseen damaged specimens.

Table 3. False Negative Rate (\%) for class "B".

\begin{tabular}{l|l|l|l}
\hline Experiment 2 & SIG & PFA & SFA \\
\hline Train: V1V2B1B2 & Test: V3B3 \\
\hline HMM & N/A & $10.67_{-3.26}^{+4.06}$ & $20.33_{-4.41}^{+5.00}$ \\
SVM & $58.33_{-5.80}^{+5.64}$ & $29.00_{-5.07}^{+5.49}$ & $62.67_{-5.74}^{+5.49}$ \\
DFFN & $82.33_{-4.80}^{+4.14}$ & $32.33_{-5.26}^{+5.61}$ & $37.00_{-5.48}^{+5.74}$ \\
CNN & $32.00_{-5.24}^{+5.60}$ & $13.00_{-3.59}^{+4.34}$ & $21.33_{-4.50}^{+5.08}$ \\
\hline Train: V1V3B1B3 Test: V2B2 \\
\hline HMM & $\mathrm{N} / \mathrm{A}$ & $4.00_{-1.92}^{+2.88}$ & $46.33_{-5.75}^{+5.82}$ \\
SVM & $79.00_{-5.05}^{+4.47}$ & $17.33_{-4.11}^{+4.77}$ & $49.67_{-5.80}^{+5.80}$ \\
DFFN & $97.67_{-2.41}^{+1.39}$ & $26.33_{-4.89}^{+5.37}$ & $43.67_{-5.69}^{+5.82}$ \\
CNN & $31.67_{-5.23}^{+5.59}$ & $2.33_{-1.39}^{+2.41}$ & $7.67_{-2.74}^{+3.62}$ \\
\hline Train: V2V3B2B3 Test: V1B1 \\
\hline HMM & $\mathrm{N} / \mathrm{A}$ & $18.69_{-4.22}^{+4.84}$ & $50.49_{-5.76}^{+5.75}$ \\
SVM & $87.87_{-4.20}^{+3.44}$ & $37.38_{-5.45}^{+5.70}$ & $83.61_{-4.64}^{+3.97}$ \\
DFFN & $87.54_{-4.24}^{+3.49}$ & $20.98_{-4.43}^{+5.01}$ & $56.07_{-5.77}^{+5.65}$ \\
CNN & $24.59_{-4.73}^{+5.23}$ & $16.07_{-3.94}^{+4.61}$ & $14.10_{-3.70}^{+4.42}$ \\
\hline
\end{tabular}

The reason is the small amount of the damaged bottles samples (only from two specimens) in the training set and the large variation in the acoustic resonance responses of the intact bottles.

Although class weighting was applied, the characteristic features of damaged bottles were not properly generalized for the unseen specimens.

McNemar's test [22] showed that there are significant differences in $\operatorname{FNR}(p \leq$ $0.05)$ across all classifier-feature combinations, except in the cases: CNN/PFA against HMM/PFA and DFFN/PFA against CNN/SIG. 
In general, the CNNs along with HMMs performed better in predicting observations from damaged bottles than other models, particularly with PFA features. In comparison with others, they managed to reliably capture the subtle differences between the damaged and intact bottles.

The CNN/PFA model achieved an overall BAR (\%) of $94.94_{-0.72}^{+0.68}$ and FNR (\%) of $9.97_{-1.34}^{+1.48}$, while the HMM/PFA achieved overall BAR (\%) of $94.36_{-0.73}^{+0.67}$ and FNR (\%) of $9.81_{-1.33}^{+1.47}$.

\section{Conclusions}

Different machine learning approaches in acoustic resonance testing of glass IV bottles are presented. Despite the rather small and imbalanced dataset with highly inconsistent classes, the presented feature analysis, and the employed classifiers achieved solid results.

The HMM and CNN models in combination with handcrafted features achieved the best overall performance in both experiments. Providing a larger amount of data, the CNNs have the potential of improving the prediction of raw signals.

In practical application, when a high detection accuracy is of critical importance, ensemble modeling should be considered. Here multiple models are created on different training sets, and the prediction is performed by aggregating their classification results.

Alternatively, higher detection performance can be achieved using one trained model and combining the predictions from multiple impacts on different positions on a tested specimen. Moreover, the classifier system should be able to detect and adapt to changing environmental conditions and data properties.

\section{References}

1. Aastroem, T.: From fifteen to two hundred NDT-methods in fifty years. In: 17th World Conference on Nondestructive Testing, pp. 25-28 (2008)

2. Abdoli, S., Cardinal, P., Koerich, A.L.: End-to-end environmental sound classification using a 1D convolutional neural network. Expert Syst. Appl. 136, 252-263 (2019)

3. Brodersen, K., et al.: The balanced accuracy and its posterior distribution. In: 20th International Conference on Pattern Recognition, pp. 3121-3124. IEEE (2010)

4. Chang, C., Lin, C.: LIBSVM: a library for support vector machines. ACM Trans. Intell. Syst. Technol. (TIST) 2(3), 27 (2011)

5. Chollet, F., et al.: Keras (2015). https://github.com/fchollet/keras

6. Clevert, D.A., Unterthiner, T., Hochreiter, S.: Fast and accurate deep network learning by exponential linear units (ELUs). arXiv preprint arXiv:1511.07289 (2015)

7. Coffey, E.: Acoustic resonance testing. In: 2012 Future of Instrumentation International Workshop (FIIW) Proceedings, pp. 1-2. IEEE (2012)

8. Cortes, C., Vapnik, V.: Support-vector networks. Mach. Learn. 20(3), 273-297 (1995). https://doi.org/10.1007/BF00994018 
9. Duchi, J., Hazan, E., Singer, Y.: Adaptive subgradient methods for online learning and stochastic optimization. J. Mach. Learn. Res. 12(Jul), 2121-2159 (2011)

10. Gokmen, G.: The defect detection in glass materials by using discrete wavelet packet transform and artificial neural network. J. Vibroeng. 16(3), 1434-1443 (2014)

11. Gunathilaka, G.: Using Fourier analysis of the resonance frequency in glassware to identify defects. In: Research Symposium on Pure and Applied Sciences. Faculty of Science, University of Kelaniya, Sri Lanka (2018)

12. Hertlin, I., Schultze, D.: Acoustic resonance testing: the upcoming volume-oriented NDT method. In: III Pan-American Conference for Nondestructive Testing (2003)

13. Hoffmann, R., Eichner, M., Wolff, M.: Analysis of verbal and nonverbal acoustic signals with the Dresden UASR system. In: Esposito, A., Faundez-Zanuy, M., Keller, E., Marinaro, M. (eds.) Verbal and Nonverbal Communication Behaviours. LNCS (LNAI), vol. 4775, pp. 200-218. Springer, Heidelberg (2007). https://doi. org/10.1007/978-3-540-76442-7_18

14. Huang, B., Ma, S., Wang, P., Wang, H., Yang, J., Guo, X., Zhang, W., Wang, H.: Research and implementation of machine vision technologies for empty bottle inspection systems. Eng. Sci. Technol. Int. J. 21(1), 159-169 (2018)

15. Liang, Q., Xiang, S., Long, J., Sun, W., Wang, Y., Zhang, D.: Real-time comprehensive glass container inspection system based on deep learning framework. Electron. Lett. 55(3), 131-132 (2019). https://doi.org/10.1049/el.2018.6934

16. Maas, A.L., Hannun, A.Y., Ng, A.Y.: Rectifier nonlinearities improve neural network acoustic models. In: Proceedings of the ICML, vol. 30, no. 1, p. 3 (2013)

17. Martín, M., et al.: Tensorflow: a system for large-scale machine learning. In: 12th USENIX Symposium on Operating Systems Design and Implementation (OSDI 16), pp. 265-283 (2016)

18. Mery, D., Medina, O.: Automated visual inspection of glass bottles using adapted median filtering. In: Campilho, A., Kamel, M. (eds.) Image Analysis and Recognition. Lecture Notes in Computer Science, vol. 3212, pp. 818-825. Springer, Heidelberg (2004). https://doi.org/10.1007/978-3-540-30126-4_99

19. Rosli, N.S., Fauadi, M., Awang, N., Noor, A.: Vision-based defects detection for glass production based on improved image processing method. J. Adv. Manuf. Technol. (JAMT) 12(1 (1)), 203-212 (2018)

20. Pedregosa, F., et al.: Scikit-learn: machine learning in Python. J. Mach. Learn. Res. 12, 2825-2830 (2011)

21. Peng, X., Li, X.: An online glass medicine bottle defect inspection method based on machine vision. Glass Technol. Eur. J. Glass Sci. Technol. A 56(3), 88-94 (2015)

22. Salzberg, S.L.: On comparing classifiers: Pitfalls to avoid and a recommended approach. Data mining and knowledge discovery 1(3), 317-328 (1997). https://doi. org/10.1023/A:1009752403260

23. Sankaran, V.: Low cost inline NDT system for internal defect detection in automotive components using Acoustic Resonance Testing. In: Proceedings of the National Seminar and Exhibition on Non Destructive Evaluation, pp. 237-239 (2011)

24. Srivastava, N., Hinton, G., Krizhevsky, A., Sutskever, I., Salakhutdinov, R.: Dropout: a simple way to prevent neural networks from overfitting. J. Mach. Learn. Res. 15(1), 1929-1958 (2014)

25. Stultz, G., Bono, R., Schiefer, M.: Fundamentals of resonant acoustic method NDT. Adv. Powder Metall. Part. Mater. 3, 11 (2005)

26. Tai, K.: The application of digital image processing technology in glass bottle crack detection system. Acta Technica 62(1A), 381-390 (2017) 
27. Tschoepe, C., Wolff, M.: Statistical classifiers for structural health monitoring. IEEE Sens. J. 9(11), 1567-1576 (2009)

28. Wawra, J.: Experiments in acoustic pattern recognition. B.Sc. thesis, Brandenburgische Technische Universität, Cottbus - Senftenberg, in German (2015)

29. Wolff, M.: dLabPro: a signal processing and acoustic pattern recognition toolbox (2014). https://github.com/matthias-wolff/dLabPro

30. Xie, L., et al.: Internal defect inspection in magnetic tile by using acoustic resonance technology. J. Sound Vib. 383, 108-123 (2016)

31. Zeiler, M.D.: ADADELTA: an adaptive learning rate method. arXiv preprint arXiv:1212.5701 (2012)

32. Zhou, X., et al.: A surface defect detection framework for glass bottle bottom using visual attention model and wavelet transform. IEEE Trans. Industr. Inf., 1 (2019). https://doi.org/10.1109/TII.2019.2935153 